\title{
Automatic Phase Segmentation of Spectrum Images
}

\author{
J. J. Friel* and R. Batcheler* \\ Princeton Gamma-Tech, C/N 863, Princeton, NJ 08542
}

Spectrum Images or Position-Tagged Files-depending on what technology was used to collect them-can be large datasets. A strategy to mine these datasets should consider, whether or not the search should take advantage of a priori knowledge. Statistical methods such as principal component analysis are called non-judgment methods and require no knowledge of the specimen composition or phase assemblage [1]. However, the method described here allows the user to specify which elements should be considered and is thus called a judgment method [2]. The segmentation of the dataset is based on the elemental maps chosen for display plus a sensitivity parameter. The user's knowledge is therefore incorporated into the segmentation strategy.

The algorithm is called recursive pixel allocation (RPA) and it consists of five passes through the dataset. These steps are: 1) Refine the average intensities and make preliminary pixel assignments. 2) Identify the phase boundaries, and reallocate indeterminate pixels. 3) Remove small phases. 4) Reduce the number of phases by combining those that are within sensitivity limits. 5) Recalculate average compositions. This process is not iterated, but the procedure can be rerun at different sensitivity levels or with different maps displayed to adjust the strategy. It should be noted that the RPA algorithm makes use of size information and eliminates small phases. If small phases are of interest, it is simple to increase the magnification to better define them. Although it is possible to generate false phases at high sensitivities, these are easily removed by lowering the sensitivity once their spectra have been examined. The output consists of a pseudocolored image of phases and a table showing intensities and area fractions. Spectra can be extracted by phase, and the compositions can be quantified based on the sum of all spectra from a phase. Position-tagged files with sufficient statistics can be collected in a minute or two at $128 \times 100$ resolution $(\sim 2 \mathrm{mb})$, and very high quality datasets can be collected in about 10 mins. at 256-200 pixel resolution $(<20 \mathrm{mb})$.

Fig. 1 shows an image and maps of an alloy consisting primarily of $\mathrm{Fe}, \mathrm{Mg}$, and $\mathrm{Si}$, plus several minor constituents. The phases in this material are easily segmented, and the output of the AutoPhase routine is shown as an image in Fig 1. Reporting the output as an image enhances the ability of the mind to perceive spatial associations and contrasts, and tabular data are also available.

Fig. 2 shows a cast iron with graphite nodules. This material has only two "true" phases; namely, graphite and iron. However, in this sample, the silicon distribution was investigated by means of a tint etch, but the results were inconclusive. By using $X$ rays and setting the AutoPhase sensitivity to the maximum, it was possible to distinguish two other distinct regions. One of these was high in silicon, and the other high in iron. In fact, the high-Fe regions are likely regions of less (thinner) etching, but the high-Si regions near the nodules appear to be real. Quantitative analysis in the bulk of the iron showed $2 \% \mathrm{Si}$; whereas, $3.9 \mathrm{wt} . \% \mathrm{Si}$ was found in the regions near the nodules.

The method of phase segmentation by recursive pixel allocation provides a measure of control of the automatic phasing process. It takes $<1 \mathrm{sec}$. to run and can sort the microstructure into phases based on the user's knowledge. A non-judgment method like Principal Component Analysis does not require this knowledge, but also cannot take advantage of it. Of course, identification of the 
elements present can also be automated. Complete manual control can be exercised by selecting representative regions and having the computer assign all pixels to one of the selected phases.

References

[1] P. G. Kotula, Spectral Imaging: Getting the Most from All that Data, this volume.

[2] F. Mosteller and J. W. Tukey, Data Analysis and Regression, Addison-Wesley, Reading, 1977

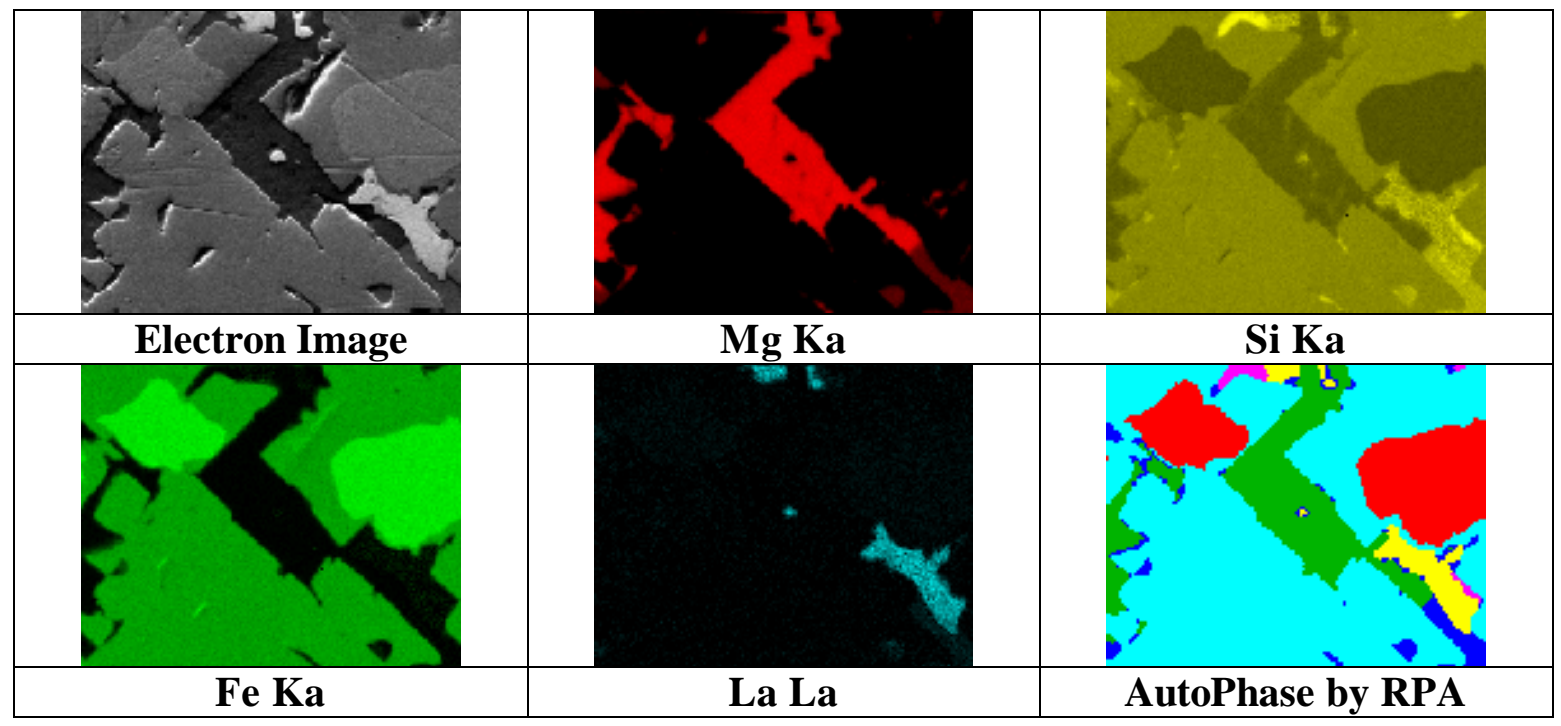

FIG. 1. Maps of an alloy of $\mathrm{Fe}, \mathrm{Mg}, \mathrm{Si}$, and $\mathrm{REE}$, showing a straightforward example of autophasing by recursive pixel allocation.

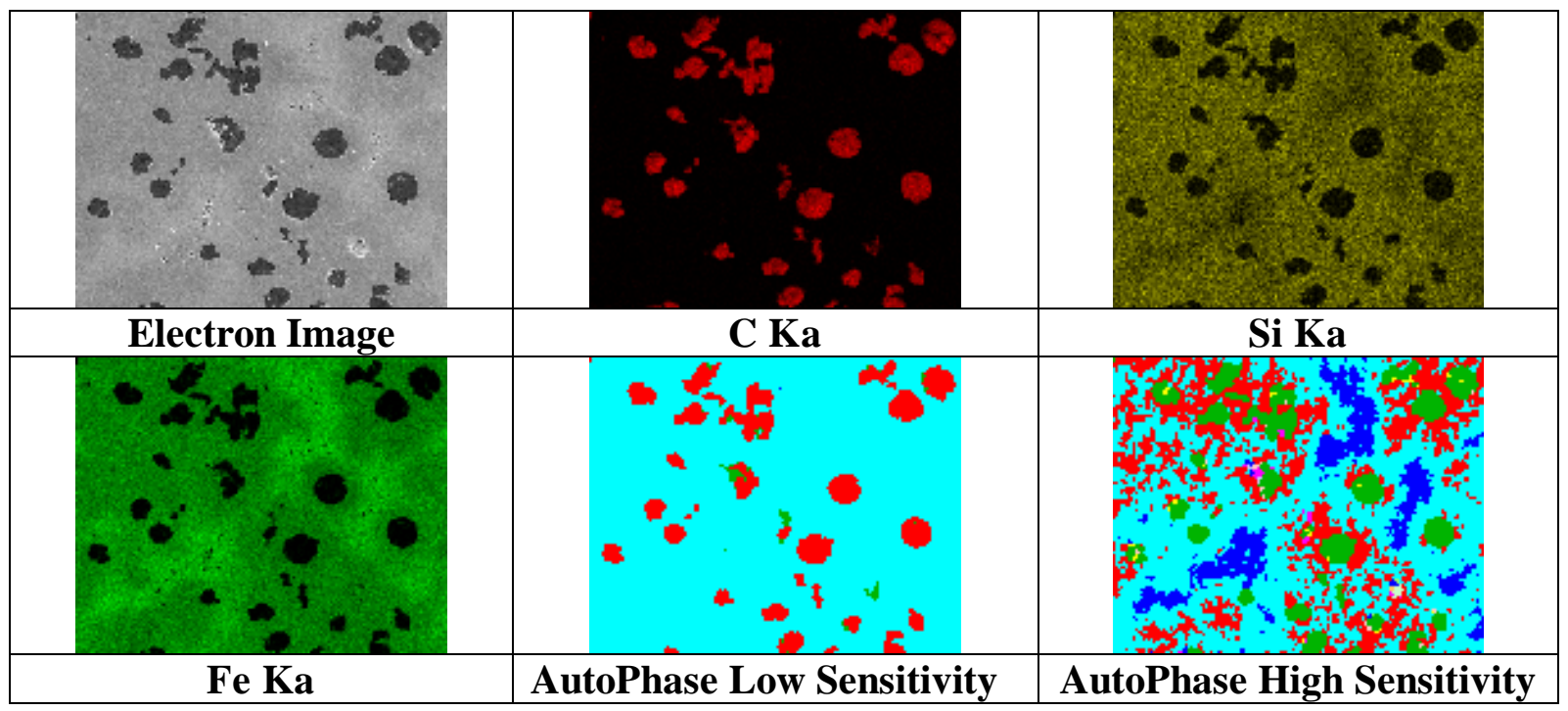

FIG. 2. Maps of a cast iron with graphite nodules. Autophase at low sensitivity shows only graphite and iron. At high sensitivity, it reveals a silicon-rich phase and an iron-rich one. 